\title{
A New Model for Prediction of the Performance of a Cappuccino Pod
}

\author{
Linhua Jiang \\ Shanghai Key Laboratory of Trustworthy Computing, East China Normal University, 200062 Shanghai, China \\ Stanford University, 269 Campus Drive, Stanford, CA 94305, USA \\ Hua Pan \\ Department of Chemical Engineering and Chemistry, Eindhoven University of Technology, Den Dolech 2, 5612 AZ \\ Eindhoven, The Netherlands \\ Haibin Cai ${ }^{*}$ \\ Shanghai Key Laboratory of Trustworthy Computing, East China Normal University, 200062 Shanghai, China \\ E-mail: hbcai@sei.ecnu.edu.cn \\ www.ecnu.edu.cn ${ }^{\ddagger}$ \\ Received 8 July 2012 \\ Accepted 21 January 2013
}

\begin{abstract}
In recent years a lot of attention and efforts have been dedicated on optimizing the design and quality of coffee pods. These would be applicable on professional as well as on household machines such as Senseo or Nestle. By providing a new method to describe the mechanisms of the brewing process within the pod, the research presented in this paper gives deeper insights into the design related aspects to improve the pods. A model incorporating the function of dynamic mass balance and the shrinking core model is employed to describe the dissolution process of the creamer in the pod. This dissolution step is the most important in determining the milky taste wanted in the case of cappuccino. The model was tested by comparing its results with those of a series of experiments. With the model presented in this paper designing better pods should be easier and more efficient.
\end{abstract}

Keywords: Cappuccino pod, creamer, shrinking core model, dynamic mass balance, dissolution process

\section{Introduction}

Because of the proven energizing and stimulating effect in humans which were discovered back in the fifteenth century [9], coffee is becoming more and more popular, up to achieving the position of the second most consumed beverage after water: it is a matter of flavor, or better still of overall sensory impact. Coffee affects millions of people's daily lives and it plays an important role in the world food market.
Although a lot of people like the flavor of coffee, they do not like the disturbing particles deriving from a bean. This fact makes beverage preparation a key step for enjoying the benefits of this product, sometimes for transforming it into a speciality even [2].

A coffee pod provides a fast and convenient manner of preparation. A coffee pod consists freshly roasted coffee, perfect ground, tampered to the correct pressure and then encapsulated by layers of filter paper. The original pod was designed by Illy Caffe over 20 years ago. In 1996 Illy opened its idea to the coffee industry

\footnotetext{
*Corresponding author. Address: Shanghai Key Laboratory of Trustworthy Computing, East China Normal University, 200062 Shanghai, China. Email: hbcai@sei.ecnu.edu.cn
} 
to bring a standard pod size to the market. The pod market has expanded rapidly in the past years with low pressure single serve coffee machines, appearing such as, Nescafe Dolce Gusto, Philips Senseo, Black \& Decker Home Cafe, Kenwood Mok Abista.

A cappuccino pod, one the most popular pods in the market, was chosen to be studied here. In a cappuccino pod, creamer is normally put at the bottom of the pod with ground coffee on the top, in between lies a layer of filter paper.

A good cappuccino must be perfectly balanced-a full bodied espresso with hints of sweetness and creaminess derived from steamed milk. In this paper, we only considered the milky taste leaving out the extraction of coffee which has been the subject of many investigations $[8,10,12]$. In other words, the main aim of the study presented is to investigate how to dissolve more creamer during the brewing process.

Recently much attention has been focused on developing new creamer formula, e.g. different skim creamer with better solubility, different combination of creamer, sugar and fat. This paper describes a new way of improving the design and quality of a cappuccino pod and gives an insight into the mechanisms of the brewing process in the pod.

We have modeled the dissolution process of the creamer in the cappuccino pod which incorporated the shrinking core model and the function of dynamic mass balance. The model was verified with experimental results. By using the model presented here, it would be easier, faster and more convenient to optimize the design of Capuccino pod. The approach we have applied is shown in Fig. 1.

\section{Process Analysis}

To have a cup of cappuccino, hot water firstly is pumped into the coffee layer, extracts the coffee, then through the filter paper and into the creamer layer where creamer particles dissolve \& disperse into the bulk liquid, finally the solution leaves the cappuccino pod and into the cup. This is so called brewing process.

Generally speaking, the brewing process of cappuccino pod includes two sub-processes: one is the extraction of coffee at the top and the other is the dissolution of creamer at the bottom. In this paper, only the second sub-process has been addressed. A theoretical model has been set up to describe the dissolution process which consisted of two parts:

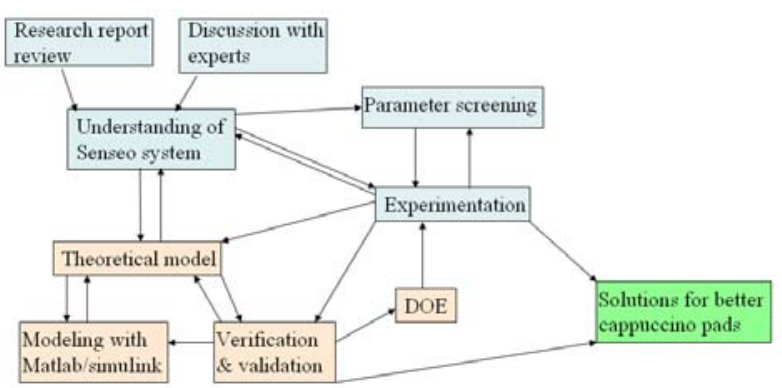

Fig. 1. The approach, it gives the outline of this study.

(a) Dynamic mass balance (continuous phase).

It takes the creamer layer as the study unit and depicts how mass of creamer balances at any time t. This is on the macro level.

(b) Shrinking core model (disperse phase).

It describes the creamer dissolution \& dispersion process on micro level.

\subsection{Dynamic mass balance (continuous phase)}

In a cappuccino pod, hot water comes from the coffee layer on top, it goes through the filter paper and reaches the creamer layer where the dissolution process takes place. During the brewing process, water comes into the pod continuously and the whole brewing process is very short, around 30 seconds. Based on this condition, it is assumed that the temperature and the creamer concentration everywhere in the pod are homogeneous at time $\mathrm{t}$.

If we take the creamer layer as the study unit, the mass balance of the creamer at any time $\mathrm{t}$ can be schematically represented as following (see Fig. 2):

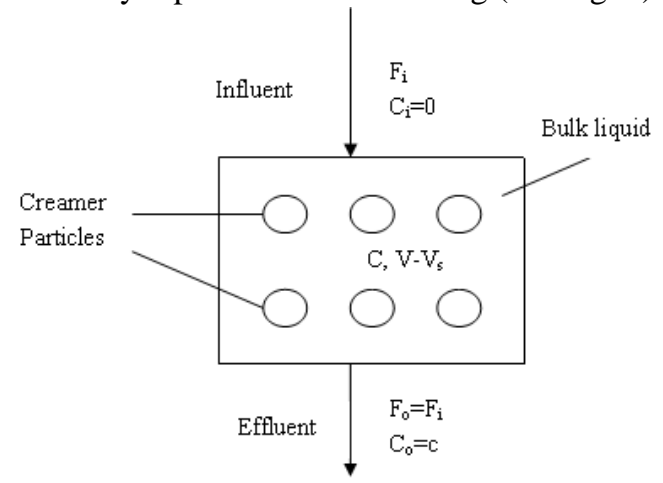

Fig. 2. Dynamic mass balance of creamer in the creamer layer at time t. 
Based on the 'law of conservation of mass', the mass into the cell equals the mass out of the cell plus the change of the mass in the cell. That is:

Amount of creamer out - Amount of creamer in $=$

Dissolved creamer from the creamer particles.

We can present this dynamic process with a mathematical equation:

$$
\frac{d M_{l}}{d t}=\rho^{*} F_{i} * C_{i}+\frac{d M_{s}}{d t}-\rho * F_{o} * c_{o}
$$

Where $M_{l}(\mathrm{~kg})$ is the amount of creamer in bulk liquid, $M_{s}(\mathrm{~kg})$ is the amount of creamer particles which has not been dissolved at time $t(s), \rho\left(\mathrm{kg} / \mathrm{m}^{3}\right)$ is the density of the bulk liquid, $F$ is flow of the bulk liquid phase $\left(\mathrm{m}^{3} / \mathrm{s}\right), C(\%)$ is the creamer concentration in the bulk liquid.

For the bulk liquid phase (continuous phase), it is assumed that the brewing process is steady, which means that the flow into and out of the cell is the same, to present in a formula: $F_{i}\left(\mathrm{~m}^{3} / \mathrm{s}\right)=F_{o}\left(\mathrm{~m}^{3} / \mathrm{s}\right)=F\left(\mathrm{~m}^{3} / \mathrm{s}\right)$.

Since there is no creamer in the down-coming liquid from the coffee pad, the amount of the incoming creamer concentration is $0 \quad\left(C_{i}(\%)=0\right)$.

Assume that liquid in the unit is ideally mixed, $C_{o}$ $(\%)=c(\%)$, where $\mathrm{c}$ is the creamer concentration in the unit. Equation (1) can now be simplified as:

$$
\frac{d M_{l}}{d t}=\frac{d M_{s}}{d t}-\rho^{*} F^{*} c
$$

And the mass of creamer is known as:

$$
M_{l}=\rho^{*} c *\left(V-V_{s}\right)=\rho^{*} c *\left(V-\frac{M_{s}}{\rho_{s}}\right)
$$

Here $V\left(\mathrm{~m}^{3}\right)$ is the volume of the creamer layer, $V_{s}\left(\mathrm{~m}^{3}\right)$ is the volume of the creamer particles, $\rho_{s}\left(\mathrm{~kg} / \mathrm{m}^{3}\right)$ is the real density of the creamer,

Take the derivative on both sides of (3) and combine with equation (1), then the final dynamic mass balance function can be described as:

$$
\left(V-\frac{M_{s}}{\rho_{s}}\right) \frac{d c}{d t}=\left(\frac{c}{\rho_{s}}-\frac{1}{\rho}\right) \frac{d M_{s}}{d t}-F^{*} c
$$

\subsection{Shrinking core model (disperse phase)}

The dissolution of solid particles in liquid can be roughly described by two processes in series: release of solute molecules from the solid surface and the diffusion of those molecules into the bulk liquid phase. The dissolution rate is determined by one of two processes or both, depending on the operation conditions [6].
The shrinking core model is used to describe situations in which solid particles are being consumed either by dissolution or reaction, and, as a result, the amount or diameters of the solid particles are "shrinking" [3]. In many cases, people applied the shrinking core model to represent the dissolution process $[5,7,11]$, and adapted the analytical expression based on different assumptions or conditions. [1] have derived an analytical expression for the variation of dissolution time as a function of the concentration of solute in the bulk liquid phase. [4] derived a model for the temporal variation of the size of a particle assuming the diffusion of solute molecules through a surface layer near the solid-liquid interface is the rate determining step.

In this study, the creamer dissolves in the water at the surface of the particle, the concentration is the saturated concentration of the creamer, which means: when $x=0, C_{b}=C_{s}$ (concentration at the boundary $C_{b}(\%)$ = saturated concentration of the creamer $C_{s}(\%), x(m)$ is the distance from the boundary of the particle). Adjacent to the surface of the particle is the boundary layer, in which the concentration of the creamer will decrease with the increased distance from the particle surface. The thickness of the boundary layer is $\delta(\mathrm{m})$, then when $x=\delta, C_{b}=c$ (c is the concentration in the bulk liquid). Outside the boundary layer is the bulk liquid, with homogeneous concentration $c$, where sufficient agitation and mixing of the liquid are assumed (See schematic Fig. 3).

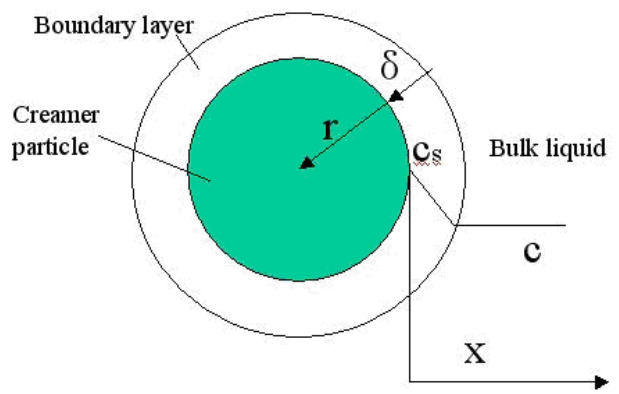

Fig. 3. The shrinking core model.

As for the creamer particles, loss of the mass is only due to the dissolution of the creamer.

$$
\frac{d M s}{d t}=\frac{d m_{s}}{d t}=-D
$$

Here, $D(\mathrm{~kg} / \mathrm{s})$ is dissolution rate, and $m_{s}(\mathrm{~kg})$ is the mass of one particle at time $t$. As for the dissolution rate of 
the creamer, it not only depends on the concentration gradient (concentration difference between the saturated concentration and the concentration in the bulk liquid, $C_{s}-c$ ), but it also depends on the contact surface area of the particles with the bulk liquid, therefore, the dissolution rate can also be described as:

$$
\frac{d M_{s}}{d t}=-k^{*} A^{*} \rho_{s}\left(C_{s}-c\right)
$$

Here $k(\mathrm{~m} / \mathrm{s})$ is diffusivity of the creamer particle, $A\left(\mathrm{~m}^{2}\right)$ is the contact surface area.

Assume all creamer particles are of spherical shape and of the same size with radius $r(m)$, the surface area can be calculated with

$$
A=n * 4 \pi r^{2}=n * 4 \pi\left(\frac{3 M_{S}}{n * 4 \pi \rho_{s}}\right)^{2 / 3}
$$

Here, $n$ is the amount of creamer particles. Then we get the final dissolution functional model:

$$
\frac{d M_{s}}{d t}=-4 k n \pi \rho_{s}\left(\frac{3 M s}{n^{*} 4 \pi \rho_{s}}\right)^{2 / 3}\left(C_{s}-c\right)
$$

From the process analysis, two equations can be derived, equation (4) is the dynamic mass balance of the creamer, equation (8) is the shrinking core model to describe the creamer dissolution process.

These two equations are coupled with each other, $M_{s}$ is a function of the time and the $c$; $c$ is also a function of the $M_{s}$ and the time. They have to be solved simultaneously before any results can be derived. And two derivatives $\left(d M_{s} / d t\right.$ and $\left.d c / d t\right)$ are also involved which makes it almost impossible to solve by hand calculation. Matlab/Simulink, developed by MathWorks, is a commercial tool for modeling, simulating and analyzing multi-domain dynamic systems. Matlab itself can facilitate the end-users to develop user-interface. Based on these considerations, it was decided to use Matlad/Simulink in this study to develop a computer program which has a core to do the simulation.

\section{Model implementation and verification}

\subsection{Model implementation}

Generally speaking, the model has two parts: the interface and the simulation core (Fig. 4 \& Fig. 5). Considering this model is intended for the use of the R\&D people who the programming is not the common tool, to make it more friendly-use and ease the work to use commands, a visual interface was designed.
With the interface, it is easier to set the values of the parameters, and also to show the results. The interface also refer to other programs to calculate for example the total amount of brew, the initial water distribution to the honeycomb and uses those data as input to the simulation part which simulates the theoretical model.

The dissolution process of creamer is a dynamic process with flow distribution, mass and concentration changing with time. The process was simulated using Simulink with the simulation results then being plotted and transferred back to the interface and shown there.

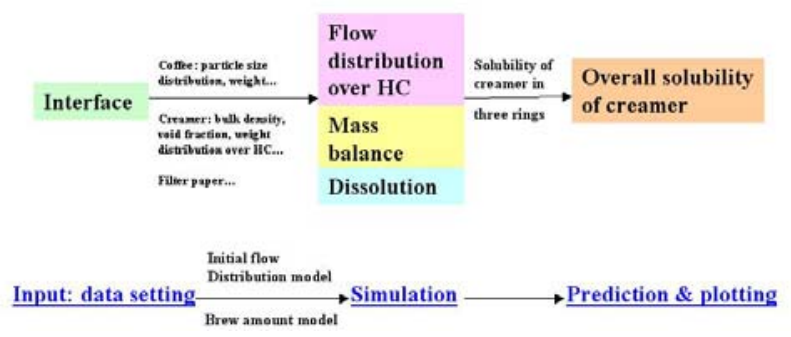

Fig. 4. Structure of the model.

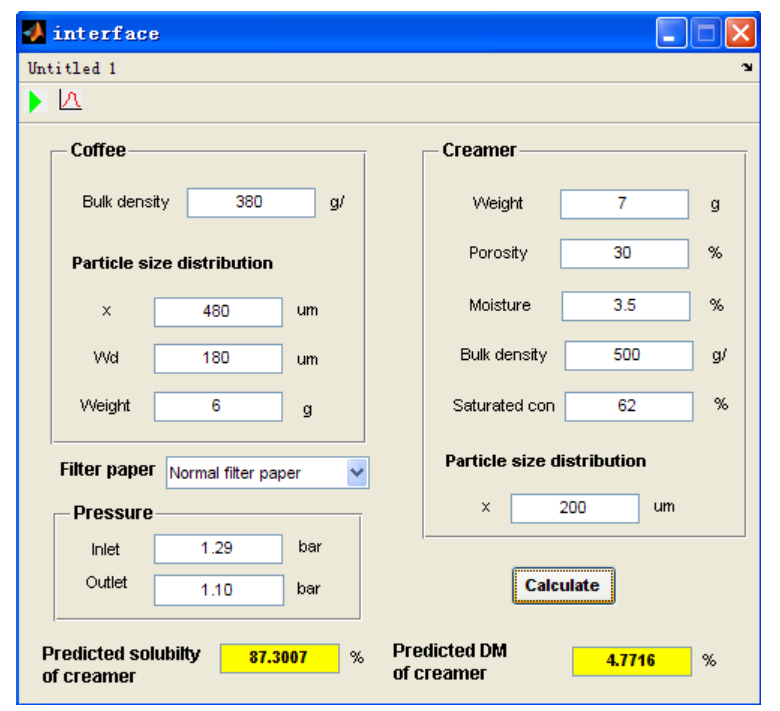

Fig. 5. Interface of the program.

This program enables the calculation of the dynamic concentration of creamer in the water in the cappuccino pod and the percentage of un-dissolved creamer (\%) versus time (s) (Fig. 6). 


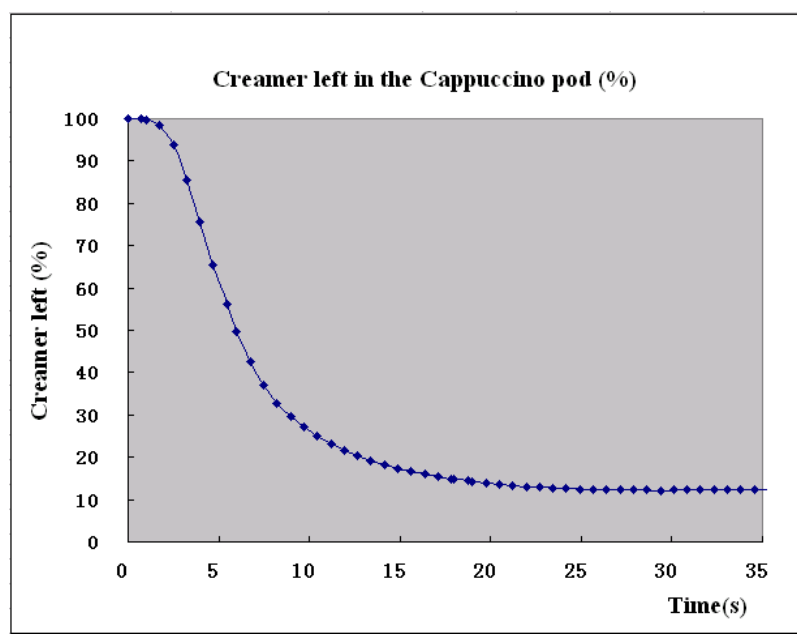

Fig. 6. Simulation result from the programmed model.

\subsection{Validation \& Verification}

Verification has been carried out all through the project. Techniques like decoupling, step-wise debugging have been used to ensure that: (a) Model has been programmed correctly; (b) The algorithms have been implemented correctly.

To validate the theoretical model, several steps have been followed as stated below:

(1) Set up an experiment design.

(2) Do the experiments according to the design.

(3) Use the experiments data for look for constant's value to make results out of the model as close to the experimental results as possible.

(4) Run the model using the parameter settings defined in the experiment design.

(5) Compare the results from (2) and (4) to see whether they are comparable or not, if not find the reasons and change the model, repeat from step (3) until the match between results from (2) and (4) is reasonably good.

As a part of the rather complicated process, dissolution of the creamer can be influenced by many factors, for example Senseo apparatus, if you change the pressure, the amount of flow or brewing time and temperature of the water, it may have impact. If you change the composition of the creamer, the structure of the honeycomb... they may also have some influence. There are still many other factors. Mind-mapping was applied to help systemize, with different colours representing factors/possible solutions at different level (Fig. 7).

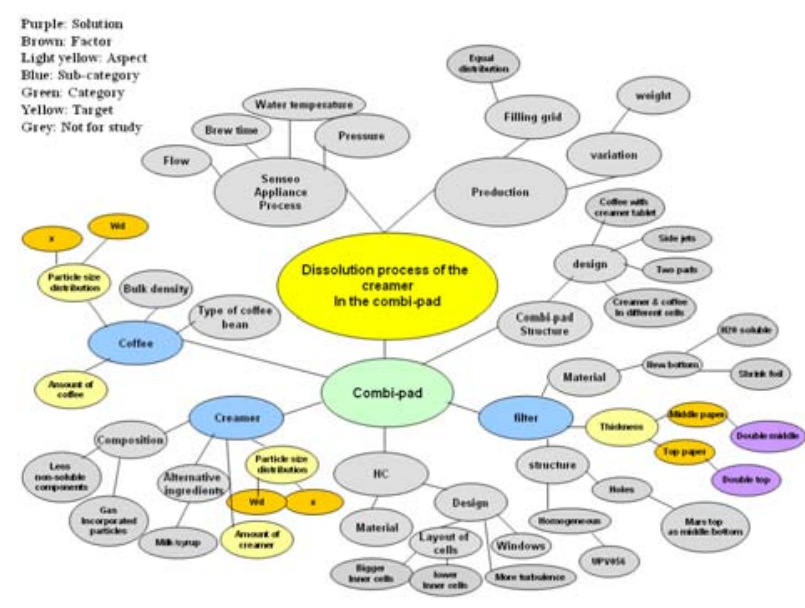

Fig. 7. Factors that can influence the dissolution of the creamer in the pad.

In the validation experiments, five parameters were selected, considering they are: (a) Not related with hardware \& recipe; (b) Measurable; (c) Controllable.

The validation range of each parameter was determined based on the possible operation window of the current production line (please see table 1)

By giving different value for each parameter, it is possible to estimate the solubility of the creamer in pod without doing real experiments. With the solubility, it is convenient to calculate the dry matter contributed by dissolved creamer in the final brew (DMcr, \%), which is closely related with the taste of the cappuccino in the cup. In this paper, $D M c r$ is used to evaluate how good the model can predict the real experiment.

To verify the model, laboratory experiments were designed by using face centered central composite design $(C C D)$. Five parameters were included, e.g. the amount of creamer added, the particle distribution of creamer. Cappuccino pods were made according to the design of the experiment. In total 56 different pods were tested and therefore got 56 real DMcr. Put the same parameter settings into the model, 56 predicted dry matter value (DMModel, \%) were calculated. To evaluate how theoretical model can represent the real experiments, DMcr and DMModel were compared in Fig. 8 (see below). A linear regression was also done to show more clearly the relationship between the $D M c r$ and DMModel. The coefficient of determination $(R-$ square) value which is a statistical measure of how well the regression line approximates the real data points was also presented. 
Table 1. Factor definition of Design of Experiment (DOE).

\begin{tabular}{|l|c|c|c|}
\hline \multicolumn{1}{|c|}{ Factors } & Min & Max & Continuous \\
\hline x50 of coffee particles(x50_coffee, $\mu \mathrm{m})(\mathrm{A})$ & 350 & 620 & Yes \\
\hline $\begin{array}{l}\text { Coffee particle size distribution width (wd_coffee, } \\
\mu \mathrm{m})(\mathrm{B})\end{array}$ & 120 & 290 & Yes \\
\hline Amount of coffee (w_coffee, g)(C) & 5.0 & 6.0 & Yes \\
\hline x50 of creamer(x50_creamer, $\mu \mathrm{m})(\mathrm{D})$ & 120 & 210 & Yes \\
\hline Amount of creamer(w_creamer, g)(E) & 6.5 & 7.5 & yes \\
\hline
\end{tabular}

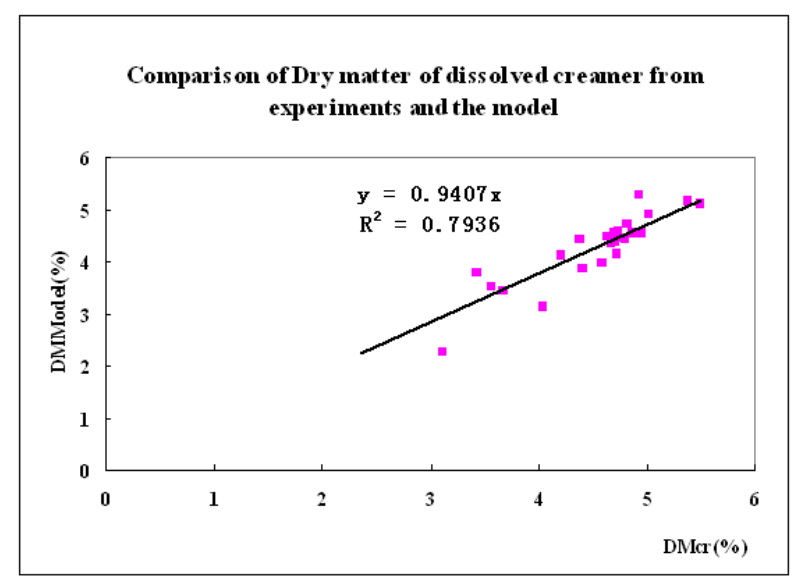

Fig. 8. Comparison of Dry matter calculated from the model (DMModel) and those from the experiments (DMcr).

In this study, there R-square is 0.7936 , which means that around $80 \%$ variation of the real experiments can be explained by the theoretical model. By using the model, it is possible to predict the dry matter contributed by dissolved creamer, which describes how well the creamer has dissolved during the dissolution process. Therefore, by applying the model presented in this study, $D M c r$ for different parameters values can be estimated without performing time and effort consuming experiments with tedious preparation steps to predict the performance of coffee pods designed.

To some extent, the higher $D M c r$ is, the more creamy taste the cappuccino will have. From the calculated $D M c r$, with statistical tool for instance Statgraphics, it is possible to find out the highest $D M c r$ and the setting values of its corresponding parameters, which could be the optimal operation values for those parameters.

\section{Conclusion and Future Works}

In this paper, a model describing the dissolution process of the creamer in a cappuccino pod is presented. It includes a shrinking core model and the function of dynamic mass balance. These two equations were coupled with each other and were solved simultaneously by a computer program using Simulink.

A series of experiments were done to examine the validity of the model. In this study, around $80 \%$ variation of the experiments can be predicted by the theoretical model.

With this model, by manipulating different parameters of the coffee and the creamer, it is possible to predict the solubility of the creamer and the dry matter of dissolved creamer without performing any effort consuming and tedious experiments. Together with statistical tool, it is possible to find out the optimal values for those operation parameters to reach the highest dry matter in the final brew. A lot of time can be saved if the model can be applied to help and facilitate the process of designing and improving a cappuccino pod.

In industry, this kind of research to set up theoretical model for optimizing process is now booming. Together with statistics tools, it can not only provide deeper insight into the fundamentals, but also help save lots of time and cost to look for the optimal operation settings, especially when the optimal is a global optimal which is not located within the normal operation ranges. With the optimal parameter settings, evaluation how these settings will influence the current production line and final product becomes also feasible, and so as to evaluate the corresponding impact on cost and profit. Therefore, without doing the pilot or real production test, based on the prediction results from the model, it is possible to provide scientific basis for the management team to decide whether or not to take the action to accept such a new design proposal.

To make the model better, some other parameters, for instance unexpected blocking of filter paper due to very fine particles, non-normal distribution of creamer particles which were not included in this model due to time limit, can be further investigated in future research. 


\section{Acknowledgements}

The authors would like to thank Dr. RAG de Graaff for revising the manuscript. This research is partially supported by the Specialized Research Fund for Doctoral Program of Higher Education of China under Grant No.20100076120011 and the Natural Science Foundation of China under Grant No. 91118008, 61170084 and 61021004. This research is also partly supported by the State Key Laboratory of Rail Traffic Control and Safety (Beijing Jiaotong University) No.RCS2011K014 and Shanghai Knowledge Service Platform for Trustworthy Internet of Things under Grant No. ZF1213.

\section{References}

1. Chen, Y. W., \& Wang, P. J.(1987). Dissolution of spherical solid particles in a stagnant fluid: an analyticle solution. The Canadian journal of chemical engineering, 67, 870-872.

2. Clarke, R.J. (2001). Coffee: Recent development. Londres (Inglaterra), Blackwell Science.

3. Fogler, H.S. (2006). Elements of chemical reaction engineering (pp.792-793). Pearson Education Inc.

4. Hsu, Jyh-ping, Lin, Da-Li, \& Lin, Mon-Jyh (1991). Dissolution of solid particles in liquids: A surface layer model. Colloids and surfaces, 61, 35-47

5. Konishi, Y., Katada, H., \& Asai, S. (1987). Kinetics of the dissolution of scheelite in aqueous Na4EDTA solutions. Metall Trans B, 18B(2), 331.

6. Sherwood, T. K., Pigford, R. L., \& Wilke, C. R.(1975). Mass transfer. New York: McGraw Hill.

7. Safaria, V., Arzpeymaa, G., Rashchib, F., \& Mostoufi, N. (2009). A shrinking particle-shrinking core model for leaching of a zinc ore containing silica. International Journal of Mineral Processing. 93(1), 79-83.

8. Sivetz, M., \& Desrosier, N.W. (1979). Coffee Technology. Westport (Estados Unidos), AVI.

9. Pendergrast, M. (1999). Uncommon grounds: The history of coffee and how it transformed our world. Basic Books.

10. Thaler, H. (1979). The chemistry of coffee extraction in relation to polysaccharides. Food chemistry, 4(1), 13-22.

11. Velardo, A., Giona, M., Adrover, A., Pagnanelli, F., \&Toro, L.(2002). Two-layer shrinking-core model: parameter estimation for the reaction order in leaching processes. Chemical Engineering Journal, 90, 231-240.

12. Vincze, I., \& Vatai, G. (2004). Application of nanofiltration for coffee extract concentration. Desalination, 162, 287-294. 